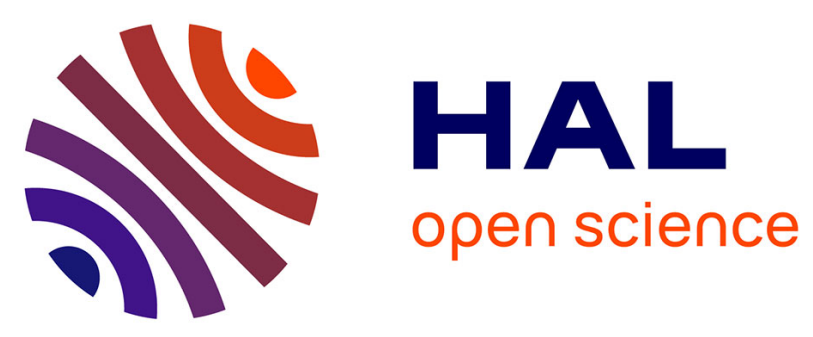

\title{
An easy-to-use technique to characterize cardiodynamics from first-return maps on $\Delta \mathbf{R R}$-intervals
}

Emeline Fresnel, Emad Yacoub, Ubiratan Freitas, Adrien Kerfourn, Valérie

Messager, Eric Mallet, Jean-François Muir, Christophe Letellier

\section{- To cite this version:}

Emeline Fresnel, Emad Yacoub, Ubiratan Freitas, Adrien Kerfourn, Valérie Messager, et al.. An easyto-use technique to characterize cardiodynamics from first-return maps on $\Delta R R$-intervals. Chaos: An Interdisciplinary Journal of Nonlinear Science, 2015, 25, pp.83111 - 83111. 10.1063/1.4928334 . hal-01672086

\section{HAL Id: hal-01672086}

\section{https://hal-normandie-univ.archives-ouvertes.fr/hal-01672086}

Submitted on 23 Dec 2017

HAL is a multi-disciplinary open access archive for the deposit and dissemination of scientific research documents, whether they are published or not. The documents may come from teaching and research institutions in France or abroad, or from public or private research centers.
L'archive ouverte pluridisciplinaire HAL, est destinée au dépôt et à la diffusion de documents scientifiques de niveau recherche, publiés ou non, émanant des établissements d'enseignement et de recherche français ou étrangers, des laboratoires publics ou privés. 


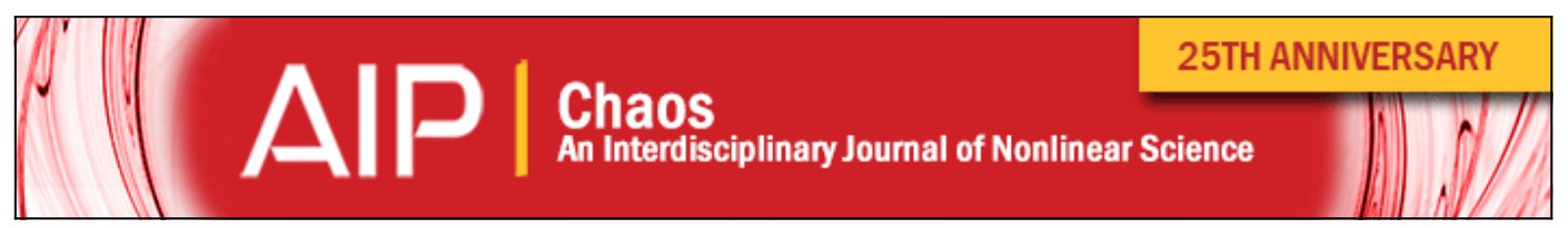

An easy-to-use technique to characterize cardiodynamics from first-return maps on $\Delta$ RR-intervals

Emeline Fresnel, Emad Yacoub, Ubiratan Freitas, Adrien Kerfourn, Valérie Messager, Eric Mallet, Jean-François Muir, and Christophe Letellier

Citation: Chaos 25, 083111 (2015); doi: 10.1063/1.4928334

View online: http://dx.doi.org/10.1063/1.4928334

View Table of Contents: http://scitation.aip.org/content/aip/journal/chaos/25/8?ver=pdfcov

Published by the AIP Publishing

\section{Articles you may be interested in}

Characterization of cardiac quiescence from retrospective cardiac computed tomography using a correlationbased phase-to-phase deviation measure

Med. Phys. 42, 983 (2015); 10.1118/1.4906246

Toward cardiac electrophysiological mapping based on micro-Tesla NMR: a novel modality for localizing the cardiac reentry

AIP Advances 2, 022156 (2012); 10.1063/1.4731801

A novel easy-to-use phantom for the determination of MTF in SPECT scanners

Med. Phys. 39, 1561 (2012); 10.1118/1.3688196

The role of M cells and the long QT syndrome in cardiac arrhythmias: Simulation studies of reentrant excitations using a detailed electrophysiological model

Chaos 14, 172 (2004); 10.1063/1.1636272

Introduction: Mapping and control of complex cardiac arrhythmias

Chaos 12, 732 (2002); 10.1063/1.1504061

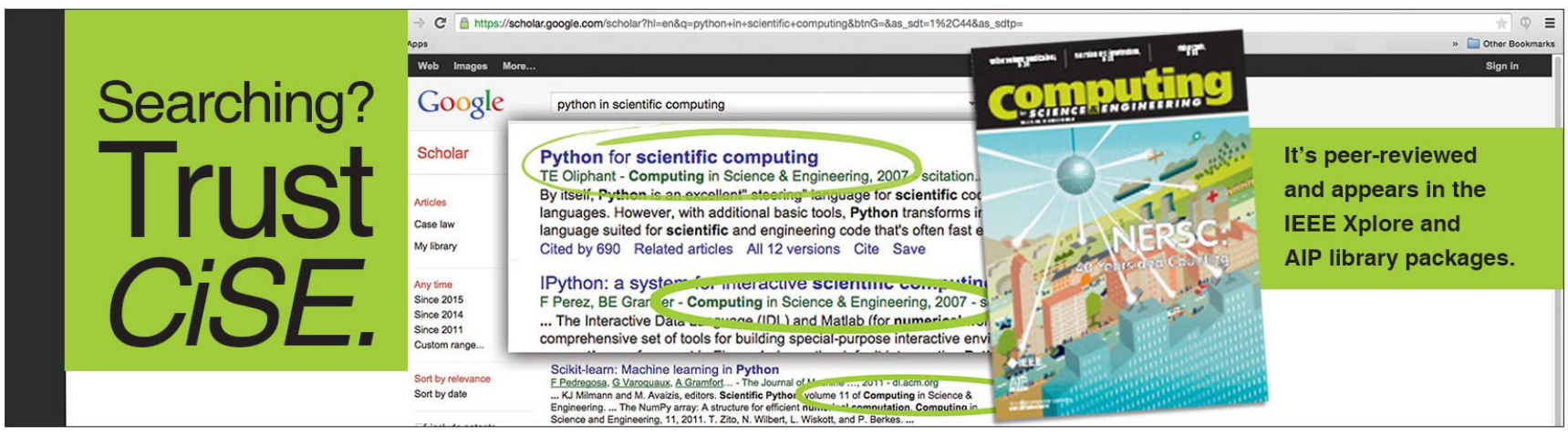




\title{
An easy-to-use technique to characterize cardiodynamics from first-return maps on $\Delta R R$-intervals
}

\author{
Emeline Fresnel, ${ }^{1,2,3, a)}$ Emad Yacoub, ${ }^{1}$ Ubiratan Freitas, ${ }^{2}$ Adrien Kerfourn, ${ }^{1,2,3}$ \\ Valérie Messager, ${ }^{1}$ Eric Mallet, ${ }^{4}$ Jean-François Muir, ${ }^{3}$ and Christophe Letellier ${ }^{1,2}$ \\ ${ }^{1}$ CORIA UMR 6614-Normandie Université, CNRS et INSA de Rouen, Campus Universitaire du Madrillet, \\ F-76800 Saint-Etienne du Rouvray, France \\ ${ }^{2}$ ADIR Association, Hôpital de Bois-Guillaume, F-76031 Rouen, France \\ ${ }^{3}$ GRHVIEA 3830, CHU Charles Nicolle, F-76031 Rouen, France \\ ${ }^{4}$ Service de pédiatrie médicale, CIC INSERM 204, CHU Charles Nicolle, F-76031 Rouen, France
}

(Received 16 December 2014; accepted 29 July 2015; published online 17 August 2015)

\begin{abstract}
Heart rate variability analysis using 24-h Holter monitoring is frequently performed to assess the cardiovascular status of a patient. The present retrospective study is based on the beat-to-beat interval variations or $\Delta R R$, which offer a better view of the underlying structures governing the cardiodynamics than the common RR-intervals. By investigating data for three groups of adults (with normal sinus rhythm, congestive heart failure, and atrial fibrillation, respectively), we showed that the first-return maps built on $\Delta \mathrm{RR}$ can be classified according to three structures: (i) a moderate central disk, (ii) a reduced central disk with well-defined segments, and (iii) a large triangular shape. These three very different structures can be distinguished by computing a Shannon entropy based on a symbolic dynamics and an asymmetry coefficient, here introduced to quantify the balance between accelerations and decelerations in the cardiac rhythm. The probability $P_{111111}$ of successive heart beats without large beat-to-beat fluctuations allows to assess the regularity of the cardiodynamics. A characteristic time scale, corresponding to the partition inducing the largest Shannon entropy, was also introduced to quantify the ability of the heart to modulate its rhythm: it was significantly different for the three structures of first-return maps. A blind validation was performed to validate the technique. (C) 2015 AIP Publishing LLC.

[http://dx.doi.org/10.1063/1.4928334]
\end{abstract}

Designing an easy-to-use technique providing a reliable characterization of the heart rate variability is of crucial importance for many clinical issues. Among the relevant properties is the complexity of the cardiodynamics, which should be neither too small nor too large, and the ability of the heart to return to its "resting" rhythm. These properties are here estimated using a Shannon entropy and an asymmetry coefficient, respectively, both of them being computed from a Poincaré map built from the variations between two successive RR-intervals. A characteristic time scale of the sinus variability, associated with the maximum Shannon entropy observed when the threshold used to construct the partition of the Poincaré map is varied, was very efficient to discriminate the different patterns we observed. This technique was designed by investigating heart rate variability on a training set of 45 adults from three patho-physiological groups and validated by blind testing on a set of 15 additional patients.

\section{INTRODUCTION}

Heart activity is driven by an electrical conduction system governed by the sinoatrial node that stimulates the myocardium. Variability of the beat-to-beat interval is a physiological feature depending on different levels of

\footnotetext{
a)Electronic mail: fresnele@coria.fr
}

regulation whose main component is the autonomic nervous system. Investigating RR interval variability allows to assess the control mechanisms and can be used as an indicator of cardiovascular health in any population. Modifications in heart rate variability reveal pathophysiological disorders and can be associated with a range of severe diseases. ${ }^{1}$ A decreased variability was found in patients with congestive heart failure $(\mathrm{CHF}),{ }^{2,3}$ left ventricular dysfunction, ${ }^{4}$ or diabetic neuropathy. ${ }^{5}$ In particular, reduced variability is a predictor of mortality in patients who underwent a myocardial infection. ${ }^{6}$

In all these works, heart rate variability has been studied using traditional time or frequency-dependent domain indicators. However, it appears that nonlinear analysis could better characterize the cardiodynamics. ${ }^{7-9}$ This can be justified since heart rate variability results from autonomic nervous system and humoral factors, which can be only described with many variables coupled in nonlinear and complex ways. ${ }^{10}$ The application of nonlinear dynamics methods to biomedical data already provided valuable information, and a prognostic value was given, for instance, using symbolic dynamics. ${ }^{8}$ Symbolic dynamics is a powerful technique to describe aperiodic behaviour and periodic orbits. It was, for instance, used by Levinson for proving the existence of aperiodic solution in a driven van der Pol equation. ${ }^{11}$ It is of a fundamental importance for describing how periodic orbits are organized within chaotic attractors. ${ }^{12,13}$ 
Our objective is not to determine whether cardiac dynamics is chaotic or not, mostly because we do not have yet a conclusive answer concerning a possible underlying determinism ${ }^{14,16}$ as it was pointed out in the challenge proposed by Glass. ${ }^{17}$ Indeed, some recent studies tend to show that cardiac variability could result from cellular stochastic processes. ${ }^{18}$ It is also influenced by respiration ${ }^{19}$ for which a first indication for the existence of an underlying determinism was recently provided in patients under mechanical ventilation. ${ }^{20}$ Such a lack of proof for a chaotic cardiodynamics does not prevent us to apply tools borrowed to the nonlinear dynamical systems theory.

The aim of this work is thus to develop an easy-to-use technique to analyze heart rate variability using 24-h Holter monitoring and to discriminate various classes of patients as challenged by Glass, ${ }^{17}$ that is, healthy subjects, patients with congestive heart failure, and patients with atrial fibrillation. After having established our procedure using a cohort of 45 patients from the Physionet database, such a technique was validated with a blind test with 15 additional patients.

\section{METHODS}

\section{A. Heart rate variability mechanisms}

Cardiodynamics is largely under the control of the autonomic nervous system, ${ }^{1,21}$ which is classically divided into two subsystems. The parasympathetic system slows the heart rate via the vagus nerve and the release of acetylcholine while the sympathetic system accelerates the pace via $\beta$ adrenegic receptors. ${ }^{22}$ Acceleration and deceleration in the heart rhythm thus result from two opposite physiological processes that can be modulated by external factors inducing short or long-term variations in the heart period. ${ }^{23}$ Their origin can be central as vasomotor and respiratory centers, or peripheral as oscillations in the arterial pressure. Other mechanisms as the circadian rhythm or short-term factors such as physical efforts, posture, and stress have an influence on the heart rate. In practice, the measured heart rate is the sum of the intrinsic heart rate and its dependence on vagal and sympathetic modulations as well as by various components. The interrelations existing between the heart rate and the vagal and sympathetic effects were for the first time quantitatively described by Rosenblueth and Simeone ${ }^{24}$ in the form

$$
\mathrm{HR}=m \cdot n \cdot \mathrm{HR}_{i},
$$

where HR is the heart rate in beats per minute (bpm), $m$ is the sympathetic influence $(m \geq 1), n$ is the vagal influence $(0<n \leq 1)$, and $\mathrm{HR}_{i}$ is the intrinsic heart rate in bpm.

Gender and age are also known to affect the heart rate variability. Many studies showed that heart rate variability is reduced when age is increased. The intrinsic heart rate was proposed by Jose and Collison ${ }^{25}$ as a function of the age $a$ (in years) as

$$
\mid \begin{array}{ll}
\mathrm{HR}_{i}=118-0.55 a & (r=-0.63) \text { for males } \\
\mathrm{HR}_{i}=119-0.61 a & (r=-0.67) \text { for females },
\end{array}
$$

where $\mathrm{HR}_{i}$ is the intrinsic heart rate in bpm. The maximal heart rate (or $\left.\mathrm{HR}_{\max }\right)$ was strongly related to age $(r=-0.90)$ according to ${ }^{26}$

$$
\mathrm{HR}_{\max }=208-0.7 a .
$$

The same linear regression was used for men and women and was not dependent on variations in individual exercise training. This reduction in the maximal heart rate could be linked to the age-related reduction in intrinsic heart rate as previously discussed. This is in agreement with the fact that older people have a vagal modulation of the heart rate smaller than younger adults. ${ }^{27}$ The reduction in heart rate variability observed among older people may result from two phenomena: a reduction in the intrinsic heart rate and in the cardio-vagal modulation.

Ageing effects in heart rate variability have been evaluated in various studies: we reported in Table I conclusions of few of them. ${ }^{28-31}$ In all studies, a significant reduction of the heart rate variability in older people as assessed by time and frequency-dependent indicators was observed. For instance, the $\mathrm{SDNN}_{\text {index }}$ is anti-correlated with age according to $^{28}$

$$
\mathrm{SDNN}_{\text {index }}=85.0-0.57 a(r=-0.63) .
$$

In particular, a reduction in vagal modulation was highlighted by a reduction in parasympathetically mediated indices of heart rate variability (RMSSD, pNN50).

Cardiodynamics was much more investigated in adults than in newborns and infants. The latter have different cardiorespiratory regulatory mechanisms. Healthy neonates present brief episodes of cardiac deceleration followed by rebound acceleration: these variations in the heart rate suggest an unstable behaviour of the servomechanism that

TABLE I. Summary of selected studies investigating the effects of ageing on heart rate variability indicators.

\begin{tabular}{lccl}
\hline \hline Reference & No. Subjects & Age range & \multicolumn{1}{c}{ Conclusions } \\
\hline 29 & 101 & $20-69$ & Time (SDNN, SDANN, SDNN index $_{\text {, RMSSD, pNN50) }}$ and frequency-domain HRV indices are anti-correlated to age \\
30 & 60 & $26-76$ & All calculated time and frequency domain HRV indices were less in older than in younger men \\
28 & 260 & $10-99$ & Time-domain HRV indices are significantly anti-correlated to ageing \\
31 & 276 & $18-71$ & Time and frequency-domain HRV indices are anti-correlated to age \\
\hline
\end{tabular}

${ }^{\mathrm{a} S D N N}$, standard deviation of all NN intervals; SDANN, standard deviation of the average NN intervals in all 5 min segments of the entire recording; $\mathrm{SDNN}_{\text {index }}$, mean of the standard deviation of all $\mathrm{NN}$ intervals for all 5 min segments of the entire recording; RMSSD, square root of the mean squared difference of successive NN intervals; pNN50, number of pairs of successive NN intervals differing by more than 50 ms divided by the total number of NN intervals. 
maintains a homeostatic level of the heart rate. The first year of life seems to be determinant in the maturation process of these mechanisms. ${ }^{32}$ During infancy, a progressive maturation of the autonomic nervous system is observed, with developmental changes of vagal and sympathetic mediation of heart rate. Finley and Nugent ${ }^{33}$ brought out this age dependency in heart rate variability by using a frequency domain study in children from 0 to 24 years. Time and frequency domain indices were found to be correlated to age in infants and children aged 3 days to 14 years. ${ }^{34}$ As we previously mentioned, these indices were found anti-correlated to the age of adults as reported in Table I.

To sum up, the autonomic nervous system is mostly responsible for the heart rate variability, which evolves during the whole life. In infancy and in older adults, there is a prevalence of the sympathetic activity over a weak vagal activity. In younger adults, the parasympathetic activity is prevailing, combined with a moderate to high level of sympathetic activity. ${ }^{35}$ The heart rate variability is thus at a low level in the early life, increases during childhood until young adulthood, and then decreases until the end of life. These features will be used in Subsection II B to define an age-dependent partition for building a symbolic dynamics.

\section{B. Nonlinear tools}

Cardiodynamics is here investigated using the differences between successive RR intervals, that is, using

$$
\Delta \mathrm{RR}_{n}=\mathrm{RR}_{n+1}-\mathrm{RR}_{n} .
$$

$\Delta \mathrm{RR}$ were first used by Tateno and Glass ${ }^{36}$ for detecting atrial fibrillation (AF) by using the density histograms of RR and $\triangle \mathrm{RR}$ intervals. A similar technique was used by Huang et al. ${ }^{37}$ Atrial fibrillation was also detected by plotting $\Delta \mathrm{RR}_{n}$ versus $\mathrm{RR}_{n}$ intervals. ${ }^{38} \Delta \mathrm{RR}$ were also used by Ritscher and Sarkar. ${ }^{39,40}$ Due to the short time series $(2 \mathrm{~min}$ ) used for plotting the first-return maps, the structures observed in Ref. 16 and in the present work were not seen in Refs. 39 and 40. First-return maps on $\Delta \mathrm{RR}$ over a long periodic window (more than one hour) were introduced in Ref. 16, where a Shannon entropy based on a partition of the first-return maps was found significantly higher in patients with atrial fibrillation. The root mean square of $\Delta \mathrm{RR}$ and a Shannon entropy computed from the RR-intervals were used for detecting atrial fibrillation in Ref. 41, quoting. ${ }^{36,40}$ In particular, they found that the Shannon entropy is significantly greater in patients with atrial fibrillation than in those with sinus rhythm as showed in Ref. 16 . Working with $\Delta \mathrm{RR}$ allows to analyze the variations in the heart rate and to overcome any long-term drift due to the sinus activity. ${ }^{16}$ We built a spatial representation of this variable by plotting first-return maps (or Poincaré maps), which consists in plotting a variable associated with the $(n+1)$ th point versus its value for the $n$th point. We built first-return maps for the whole data set (at least many hours in each case) as it was done for computing first-return map from RR-intervals in Ref. 42 or from $\Delta \mathrm{RR}$ in Ref. 16.

For healthy adults, these maps present a circular cloud of points centered around zero and associated with a low variability, which is typically associated with the sinus variability. Isolated points distant from the first bisecting line are representative of large beat-to-beat variations in the heart rate, thus corresponding to arrhythmias. In a previous study, ${ }^{16}$ we showed that first-return maps present specific structures that could be summarized as follows. A small disk centered at the origin can be seen for healthy people, four segments for people with ventricular premature contractions (VPCs) and a triangular shape for patients suffering from atrial fibrillation. As we will discuss in Section III, these structures could be considered as the main architectures encountered in many situations.

In order to characterize them, we started by computing the angular distribution of points located out of the central disk, as

$$
\theta_{n}=\arg \left(\Delta \mathrm{RR}_{n}+i \Delta \mathrm{RR}_{n+1}\right)
$$

Distribution of angles $\theta_{n}$ helped us to clearly specify the characteristics of these main structures we observed in firstreturn maps. This angular distribution allows to numerically assess the shape of first-return maps. However, the time sampling of the ECG implies a quantization of the RR intervals, which is also present in the $\Delta R R$. This quantization of the space defined by the axes $\Delta \mathrm{RR}_{n}$ and $\Delta \mathrm{RR}_{n+1}$ causes an artifact in the distribution of the angles, the samples being predominantly grouped in a few specific angles. In order to avoid that, we added a random noise to each $\Delta \mathrm{RR}$ time series, taken from a uniform distribution bounded inside the quantization intervals, such as the re-quantization of the modified series is identical to that performed on the initial data. The resulting quantization step after noise addition is much smaller, that is in the order of the computer precision. Moreover, only the points located at a distance greater than $100 \mathrm{~ms}$ from the center of the first-return maps were considered, in order to highlight the variations in the heart rate that are not induced by the sinus node. It also prevents from a substantial variability of the computed angles since the points that are close to the center of the maps are more likely to induce great angular variations under the noise influence.

The heart dynamics is investigated using a symbolic dynamics based on a partition of first-return maps into domains chosen for distinguishing normal variability from arrhythmias. As we discussed earlier, the heart rate variability is related to age and so the size of the central disk associated with sinus variability. The range visited by $\Delta \mathrm{RR}_{n}$ is split into three domains according to the partition

$$
\sigma_{n}=\mid \begin{array}{lll}
0 & \Delta \mathrm{RR}_{n} \leq-\tau \\
1 & \text { if } \quad & -\tau<\Delta \mathrm{RR}_{n}<+\tau \\
2 & & \Delta \mathrm{RR}_{n} \geq+\tau,
\end{array}
$$

where symbol 1 represents small variations due to the sinus rhythm (the central disk), symbol 0 is associated with fast decreasing of RR intervals, and symbol 2 corresponds to fast increasing of RR intervals. Such a symbolic dynamics thus transforms series of real numbers into symbol sequences that are easier to treat and interpret. ${ }^{16}$ 
In order to better distinguish different groups of patients with the same age but with different diseases, the partition should be only age dependent. Such a dependency should be related to those demonstrated in Eqs. (2), (3), and (4) and to the fact that variability is developed during infancy. According to the slope of the curve for the heart rate index or the $\mathrm{SDNN}_{\text {index }}$ versus age, it is possible to choose a linear dependency for adults as

$$
\tau(a)=89-0.60 a,
$$

where $a$ is the patient age in years and $\tau$ is the "radius" of the central disk in milliseconds. The coefficient " 0.60 " corresponds to a rough approximation of the slope of various curves versus age $a$ as discussed in Section II A (see Eqs. (2), (3), and (4)). The intercept value was determined by trial-and-errors for providing a good discrimination between patients with various diseases. This value can be varied a little without significantly affecting the results. Based on a preliminary investigation of the heart dynamics recorded in newborns, we found that the threshold should be around $25 \mathrm{~ms}$ for few month newborns. This assertion was confirmed by measures found in the literature. ${ }^{15}$ The maximal variability, and consequently the maximal threshold $\tau$, being assumed to be observed at about 20 years, we constructed a dependency of $\tau$ versus the age according to an exponential function

$$
\tau(a)=\left(1-\exp \left(-\frac{a}{\tau_{1}}\right)\right)\left(89-\tau_{0}-0.60 a\right)+\tau_{0},
$$

where $\tau_{1}$ is a time constant equal to 7 years and $\tau_{0}$ represents the initial threshold, that is $25 \mathrm{~ms}$ for the newborn infants. When only adults are considered, the linear regression (8) is sufficient to determine the threshold $\tau$, as the exponential function converges towards the linear curve designed for adults.

Once the symbolic dynamics is built according to partition (7), the heart variability is investigated in terms of subsequences of a given number $N_{q}$ of symbols. We used $N_{q}=6$ consecutive symbols, taken among the $N_{p}=3$ different symbols $\{0,1,2\}$. This leads to $N_{p}^{N_{q}}=3^{6}=729$ possible sequences. Our statistics is thus correctly defined since a $24-\mathrm{h}$ Holter contains about 100000 RR intervals. From these symbolic sequences, we choose to characterize the cardiodynamics by computing a Shannon entropy that was introduced to measure the information production rate of a process ${ }^{43}$ and already used in Ref. 16. We are here using such an entropy as a measure of the "complexity" of the dynamics, a small entropy being associated with a simple (periodic) solution and a large one with a complex (aperiodic) solution. Typically, the entropy for a period 1 regime is equal to zero since there is no information produced from an event to the next one. Contrary to this, the entropy is maximum when all possible sequences (distinguished states) are equiprobable. The Shannon entropy is defined as

$$
\tilde{S}_{h}=-\sum_{n=0}^{N_{p}^{N_{q}}-1} P_{n} \log P_{n},
$$

where $P_{n}$ is the realization probability of the $n$th symbolic sequence among the 729 possible ones where $n$ corresponds to the integer of the 6-symbol sequence read in base 3 . The Shannon entropy is normalized by the largest entropy, which is obtained when all sequences are equiprobable with a probability

$$
P_{n}=\frac{1}{N_{p}^{N_{q}}} \quad(\forall n)
$$

we thus have

$$
\begin{aligned}
S_{\max } & =-\sum_{n=0}^{N_{p}^{N_{q}}-1} \frac{1}{N_{p} N_{q}} \log \frac{1}{N_{p}^{N_{q}}} \\
& =N_{q} \log N_{p}=6 \log 3=6.59 .
\end{aligned}
$$

The relative Shannon entropy $S_{h}=\frac{\tilde{S}_{h}}{S_{\max }}$ is thus within the interval $[0,1]$. A relative Shannon entropy $S_{h}$ close to 1 implies a very complex dynamics for which arrhythmias are frequent. By definition, sinus rhythm is mostly associated with small variations (symbols "1") and characterized by an entropy close to 0 . In order to distinguish patients with a too small variability (realizing almost only sequences "111111") from the normal ones, the threshold $\tau$ is thus small enough to induce entropy significantly greater than 0 for normal subjects. As we will see in Section III, the entropy for subjects with a normal sinus rhythm (NSR) is roughly 0.3. This means that, if we consider that the 40 sequences containing only isolated symbols " 0 " and/or "2" (i.e., they are between two symbols " 1 ") are equiprobable and that each one represents around $1 \%$ of the realized sequences (the others being the sequence "111111"), we have

$$
S_{\text {norm }}=\frac{-1}{6.59}\left(\frac{60}{100} \log \frac{60}{100}+40 \times \frac{1}{100} \log \frac{1}{100}\right)=0.33 .
$$

A "normal" cardiodynamics is therefore associated with roughly $30 \%$ of isolated fast variations, according to our approach.

The symbolic dynamics defined by partition (7) splits the first-return map into 9 panels (Fig. 1), each of them being characterized by a probability of visits $\eta_{i j}$, where $i$ is the symbol assigned to $\Delta \mathrm{RR}_{n}$ and $j$ is the one assigned to $\Delta \mathrm{RR}_{n+1}$. The central domain designated by 11 therefore corresponds to a cardiac dynamics ruled by the sinus node.

Asymmetry in the sinus heart rate was already investigated using different measures from the RR intervals. ${ }^{4-46}$ In the present study, we choose to use the differences $\Delta R R$ between successive RR intervals; we therefore did not perform our analysis from the same variable. Moreover, we did not filter the data to remove arrhythmias (such as VPCs), contrary to what is commonly done in other studies ${ }^{8,47}$ Our objective was thus to define an index being representative of the asymmetry of the entire cardiac dynamics, including arrhythmias if there are any. We defined our asymmetry coefficient as 


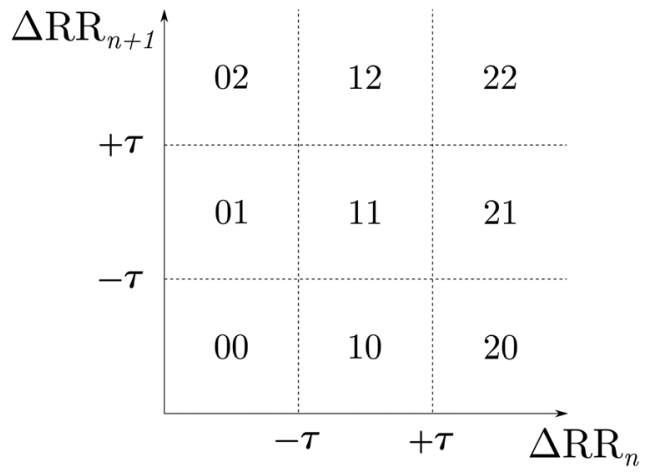

FIG. 1. Partition of the first-return map in 9 panels, according to partition (7) used to define the symbolic dynamics.

$$
\alpha=\frac{1}{4}\left[\frac{\eta_{00}}{\eta_{22}}+\frac{\eta_{01}}{\eta_{21}}+\frac{\eta_{02}}{\eta_{20}}+\frac{\eta_{10}}{\eta_{12}}\right],
$$

where each term is the ratio between the probability of visits of a domain corresponding to fast accelerations and its symmetric under an inversion symmetry (in the plane of the firstreturn map on $\triangle \mathrm{RR})$. Indeed, domains containing at least one symbol " 0 " $(00,01,10)$ correspond to the emergence of a fast decreasing between two consecutive RR intervals (acceleration in the heart rate), whereas domains containing at least one symbol " 2 " $(12,21,22)$ correspond to the emergence of a fast increasing between two consecutive RR intervals (deceleration in the heart rate). Domains 02 and 20 containing at the same time the two symbols " 0 " and " 2 ", we considered the first emergence of a " 0 " to characterize a fast acceleration event.

Consequently, an $\alpha$ coefficient roughly equals to 1 is representative of a balance between fast accelerations and decelerations in the rhythm. $\alpha<1$ represents a tendency to fast decelerations and $\alpha>1$ represents a tendency to fast accelerations in the cardiac rhythm.

For each patient, we plotted the asymmetry coefficient $\alpha$ versus the Shannon entropy $S_{h}$ and found that patients are thus grouped in different domains according to the structure of their first-return maps. These two biomarkers thus allow to automatically detect the structure of the first-return maps on $\Delta \mathrm{RR}$. The structures of the first-return map do not reveal by themselves how often they are visited or not. The probability $P_{111111}$ of realization of six consecutive beats with a limited variability (less than $\pm \tau$ ) can be used for assessing how often is visited the central disk and, indirectly, how frequent are arrhythmias (here considered as any beat-to-beat fluctuations exceeding $\pm \tau$ ).

To complete our set of biomarkers, we also introduced a characteristic time scale of the cardiac dynamics obtained as follows. We first introduced a symbolic dynamics defined according to the partition

$$
\sigma_{n}=\mid \begin{array}{lll}
0 & \text { if } & \left|\Delta \mathrm{RR}_{n}\right|<\tau_{\text {var }} \\
1 & \text { if } & \left|\Delta \mathrm{RR}_{n}\right| \geq \tau_{\text {var }},
\end{array}
$$

where $\tau_{\text {var }}$ is a time threshold that was varied from 0 to $200 \mathrm{~ms}$. Such a symbolic dynamics with $N_{p}=2$ symbols was used with $\tau=10 \mathrm{~ms}$ in order to define some indices for detecting low variability and decreased heart rate variabilities. ${ }^{9,48}$ Instead of selecting a unique given value, we varied the time threshold $\tau_{\text {var }}$. From the $N_{p}^{N_{q}}=2^{6}=64$ possible sequences of length $N_{q}=6$ constructed with $N_{p}=2$ symbols, we computed the Shannon entropy for each threshold $\tau_{v a r}$. The value $T_{c}$ of $\tau_{v a r}$ corresponding to the maximum Shannon entropy was retained as a characteristic time scale of the cardiac dynamics. We do not have yet a physiological meaning to propose for such a time scale but it was retained due to its discrimination power.

\section{APPLICATION TO TRAINING DATA SETS AND BLIND VALIDATION}

\section{A. PHYSIONET data}

In order to test the discriminating power of our technique, three different groups of patients from the Physionet database $^{49}$ were selected. For each of them, a long-term ECG signal (24 h) containing around $100000 \mathrm{RR}$ intervals was downloaded. The first group included 15 healthy subjects with NSR and no significant arrhythmias, aged $34 \pm 7$ years (range 20 to 45 ). According to Eq. (8), the corresponding threshold $\tau_{\mathrm{NSR}}$ required for constructing the symbolic dynamics was calculated for each patient (the average value was around $69 \mathrm{~ms}$ ). The second group was made of 15 patients with severe CHF (NYHA class 3-4), aged $58 \pm 6$ years (range 48 to 71 ). The corresponding threshold $\tau_{\mathrm{CHF}}$ was calculated for each patient (the average value was around $54 \mathrm{~ms}$ ). The third group contained 15 patients with sustained AF (one or more episodes during at least $20 \mathrm{~h}$ of the recordings), aged $66 \pm 12$ years (range 39 to 87 ). Individual age of each patient was not specified in the database, so we put the same time threshold $\tau_{\mathrm{AF}}$ for all the patients in this group, that is, $50 \mathrm{~ms}$ corresponding to the mean age according to Eq. (8).

\section{B. Structures of the first-return maps}

First, first-return maps from the $\Delta \mathrm{RR}$ variable were plotted for all patients from the three groups. The distributions of the angles $\theta_{n}$ exhibit significant departures between the three groups of patients. First-return maps of healthy subjects are characterized by a central cloud of points whose size depends on the considered patient. Some points can be out of the central cloud but their repartition is rather homogeneous as confirmed by the rather uniform angular distribution (Fig. $2(\mathrm{c})$ ). The angles around $\frac{\pi}{2}$ and $\frac{15 \pi}{8}$ are slightly more often observed. They correspond to points located in panels 12 and 21 (Fig. 1), respectively, corresponding to isolated decelerations in the heart rate followed by a return to the central disk, suggesting the influence of the parasympathetic system, which mainly slows the pace. This provides evidence for the hypothesis that normal subjects present fast and isolated variations in the heart rate.

Patients affected by congestive heart failure are characterized by a central cloud (abnormally smaller than in healthy subjects) and four well-defined segments whose orientations do not depend on the patients. Typical angular distribution for such patients shows that four segments are 


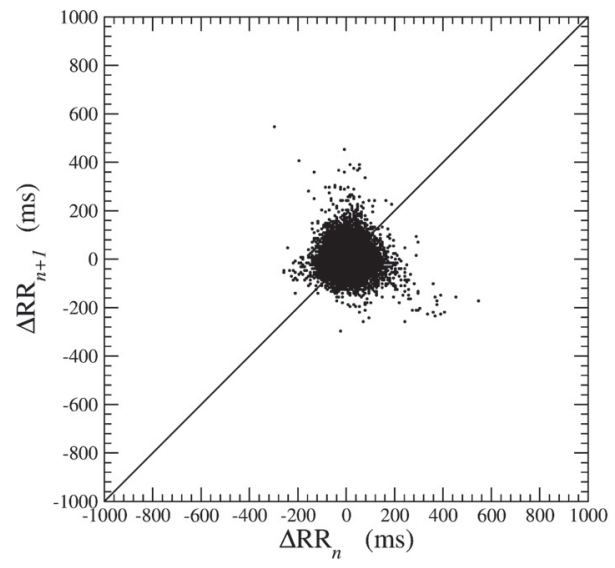

(a) $\mathrm{NSR}_{13}$ patient

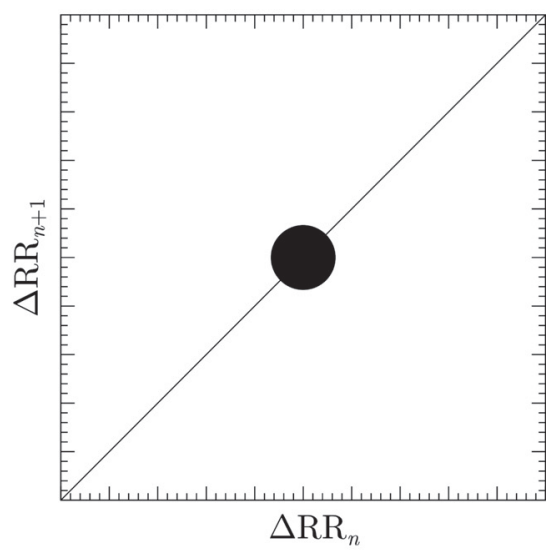

(b)Healthy model

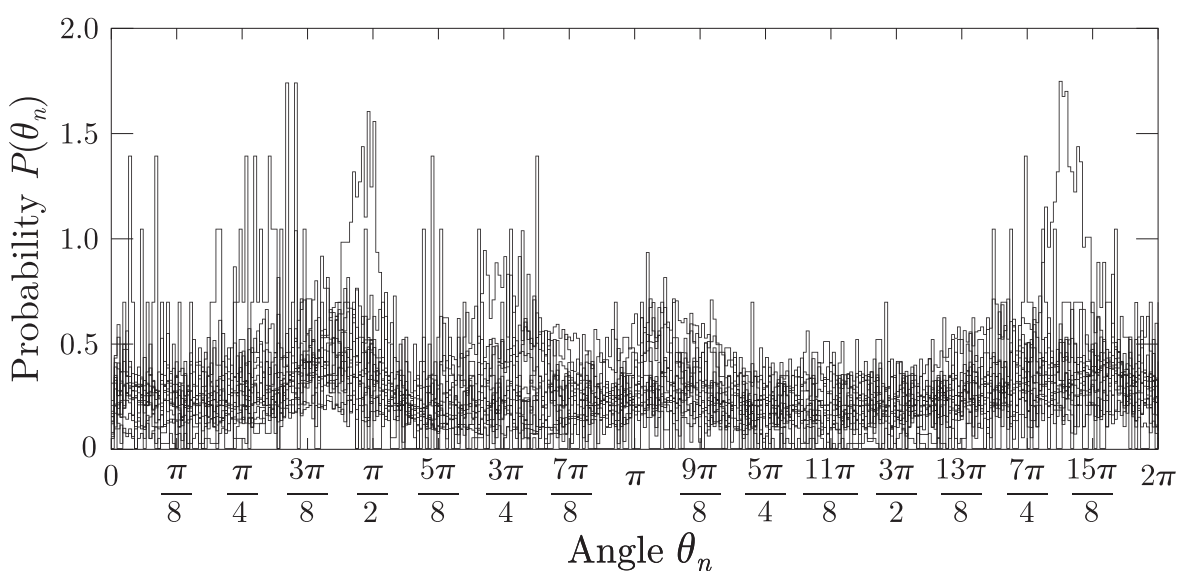

(c)Angular distribution for the 15 patients of the NSR group

FIG. 2. First-return map built on $\Delta R R$ for a Physionet patient from the NSR group and its corresponding schematic model, built from the angular distribution. oriented at $\frac{5 \pi}{8}, \pi, \frac{3 \pi}{2}$, and $\frac{15 \pi}{8}$ (Fig. 3). These segments correspond to VPCs consisting in a premature contraction, which is not triggered by the sinus node but by an ectopic center: they may occur randomly. The premature beat is usually followed by a prolonged beat that offsets the anticipation in order to synchronize cardiac contraction with the sinus node activity. ${ }^{50}$ The RR intervals take the following successive values:

$$
\ldots T T T(T-\delta t)(T+\delta t) T T \ldots,
$$

where $T$ is the mean duration of a cardiac beat and $\delta t$ is the delay by which the first beat is anticipated. The corresponding $\triangle \mathrm{RR}$ are thus

$$
\ldots 00-\delta t+2 \delta t-\delta t 0 \ldots
$$

As schematized on Fig. 3(b), segment A corresponds to the occurrence of the acceleration by $-\delta t$. Segment B has a slope -2 , that is, oriented at $\frac{5 \pi}{8}$, and corresponds to the transition from $-\delta t$ to $+2 \delta t$. Segment $\mathrm{C}$ corresponds to the transition from $+2 \delta t$ to $-\delta t$ and has a slope $-\frac{1}{2}$, that is, oriented at $\frac{15 \pi}{8}$. Segment D corresponds to the return to the central disk. The associated symbolic sequence is therefore

$$
\begin{array}{lllllll}
\ldots 1 & 1 & 0 & 2 & 0 & 1 \ldots
\end{array}
$$

Four domains of the first-return map are therefore successively visited during a VPC event: panels 10, 02, 20, and 01 as confirmed by the angular distribution (Fig. 3(c)). The structure of the first-return maps of patients with congestive heart failure thus suggests that they are subject to VPCs. A relation between heart failure and VPCs had been established but the underlying mechanism remains poorly understood. ${ }^{51}$ In practice, not all VPCs are followed by a complete compensatory pause and other symbolic sequences may therefore appear. They were not taken into account in this work.

When bursts of successive VPCs occur, two additional segments can be distinguished in the first-return map at $\theta=$ $\frac{3 \pi}{4}$ and $\theta=\frac{7 \pi}{4}$, respectively. The corresponding symbolic sequence is

$$
\ldots 110(20)^{p} 1 \ldots
$$

where $p$ is the number of successive VPCs (the sequence " 20 " is repeated $p$ times).

Patients affected by atrial fibrillation present a very wide and dense first-return map, with a characteristic triangular shape (Figs. 4(a) and 4(b)). The central disk cannot be distinguished: the cardiac rhythm is not governed by the sinus node. This triangular shape is highlighted by the angular distribution, which presents three main orientations located at $\frac{\pi}{2}, \pi$, and $\frac{7 \pi}{4}$, respectively (Fig. 4(c)). Contrary to what is observed in angular distributions of healthy subjects, for which the angles were uniformly distributed, or in patients with congestive heart failure, for which points with 


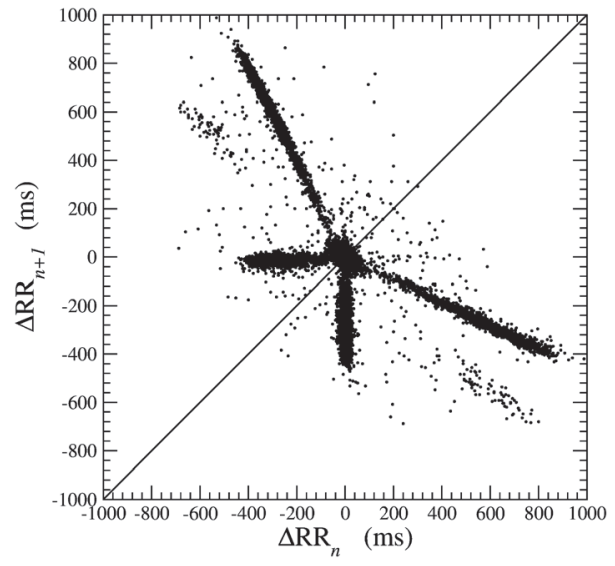

(a) $\mathrm{CHF}_{7}$ patient

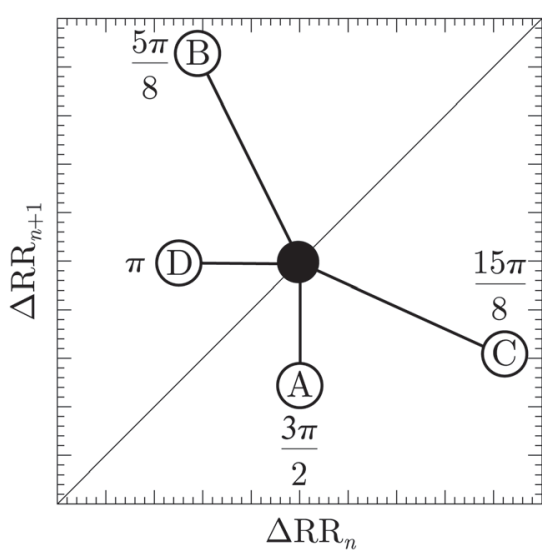

(b)CHF model

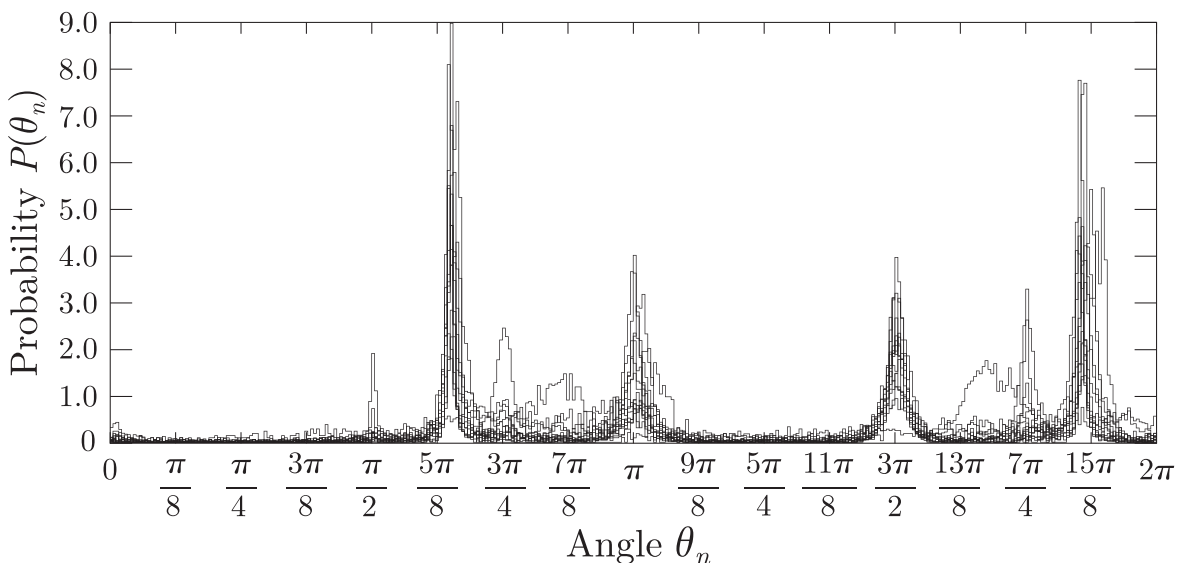

(c)Angular distribution for the 15 patients of the CHF group

FIG. 3. First-return map built on $\Delta R R$ for a Physionet patient from the $\mathrm{CHF}$ group and its corresponding schematic model, built from the angular distribution. specific angles were mostly distributed among sharp peaks, large intervals of angles were visited for patients with atrial fibrillation, evidencing the great disorder in their cardiodynamics. Angles between $\frac{\pi}{2}$ and $\pi$ are uniformly distributed and shape the base of the triangle, whereas the peak located around $\frac{7 \pi}{4}$ is responsible for the apex. Points along the three medians of the triangle mostly correspond to fast isolated decelerations in the heart rate. When a deceleration event occurs with an elongated RR interval, the heart returns to its normal activity by a resynchronization with the sinus node during the following beat. In that case, RR intervals take the successive values

$$
\ldots T T T(T+\delta t) T T T \ldots
$$

The corresponding $\Delta \mathrm{RR}$ are thus

$$
\ldots 00+\delta t-\delta t 00 \ldots
$$

and the associated symbolic sequence is

$$
\text { ... } 112011 \ldots
$$

Three panels of the first-return map are successively visited during this type of arrhythmias (panels 12, 02, and 01) as highlighted by the angular distribution. Atrial fibrillation results from an uncoordinated action of the atrial myocardial cells leading to rapid and irregular contractions of the atria.
Consequently, short irregular heart beats (tachycardia) frequently occur in these patients. Such an acceleration of the heart rate is balanced according to a property of the atrioventricular node, which presents a decremental conduction, that is the more frequently the node is stimulated, the slower it conducts. ${ }^{52}$ This prevents rapid conduction to the ventricles in the case of rapid atrial rhythms as encountered in atrial fibrillation. These patients suffering from atrial fibrillation are thus subject to an interplay between very frequent fast accelerations and decelerations in the heart rhythm, decelerations structuring the triangular shape of the first-return maps.

\section{Results}

A specific structure for the first-return maps was highlighted for each of the three groups of patients. These maps can be regrouped according to their structure by using the Shannon entropy $S_{h}$ and the asymmetry coefficient $\alpha$ as shown in Fig. 5. Typically, the $S_{h}-\alpha$ plot can be divided into three panels by using the two thresholds $S_{\mathrm{c}}=2 S_{\text {norm }}$ and $\alpha_{\mathrm{c}}=1.5$ as follows.

- panel NSR if $S_{h}<S_{\mathrm{c}}$ and $\alpha<\alpha_{\mathrm{c}}$;

- panel CHF if $\alpha \geq \alpha_{\mathrm{c}}$;

- panel AF if $S_{h} \geq S_{\mathrm{c}}$ and $\alpha<\alpha_{\mathrm{c}}$.

Each of these panels corresponds to one specific group of patients, which can therefore be discriminated by using 


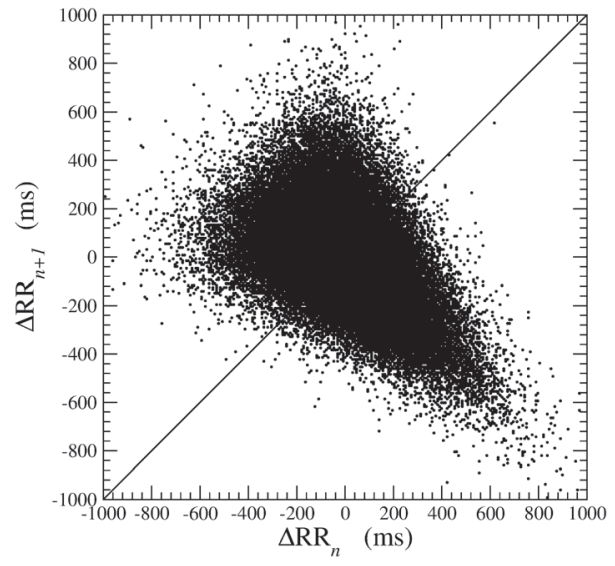

(a) $\mathrm{AF}_{13}$ patient

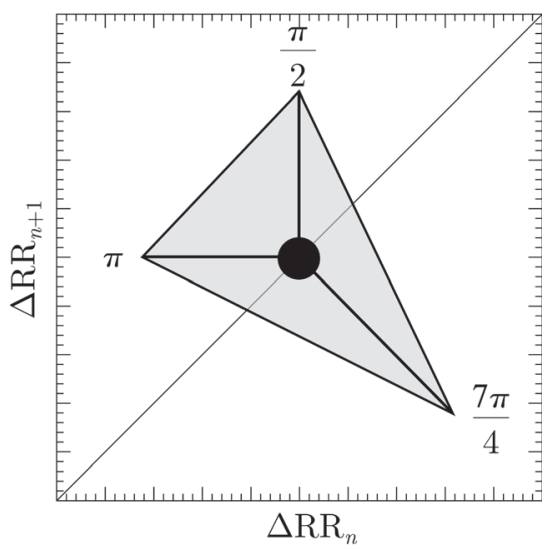

(b)AF model

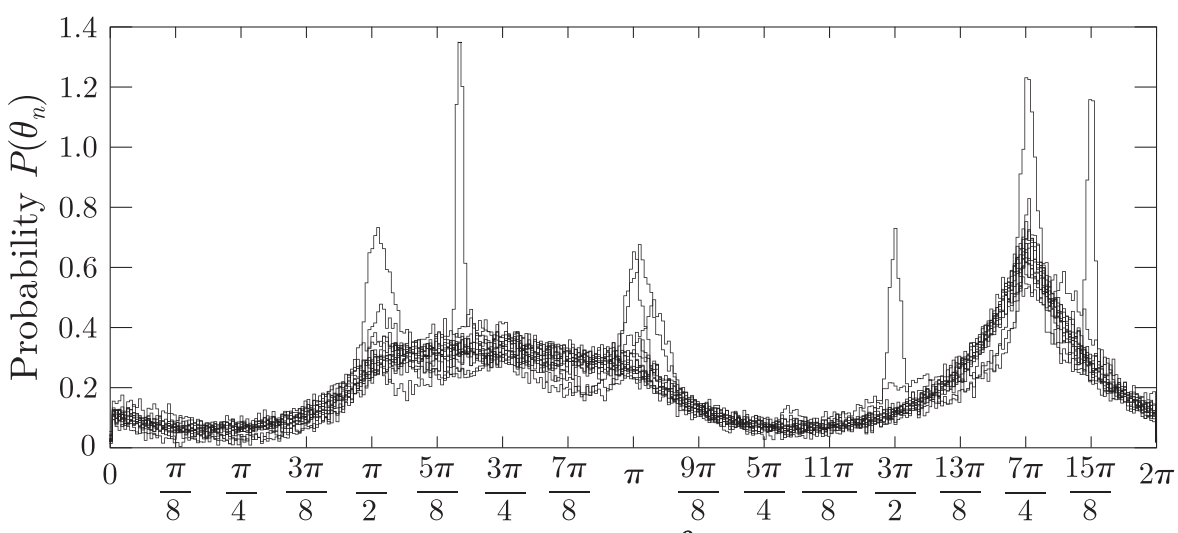

Angle $\theta_{n}$

FIG. 4. First-return map built on $\Delta R R$ for a Physionet patient from the $\mathrm{AF}$ group and its corresponding schematic model, built from the angular distribution.

\section{(c)Angular distribution for the 15 patients of the AF group}

these two biomarkers. CHF patients whose first-return maps present characteristic segments due to the occurrence of VPCs are located in the CHF-panel (left top part of Fig. 5). First-return maps with a triangular shape correspond to the largest Shannon entropies (right part of Fig. 5), thus revealing complex dynamics, and are associated with patients affected by atrial fibrillation. First-return maps (mostly appearing as a central disk) corresponding to patients with normal sinus rhythm are in the NSR-panel (left bottom part of the map shown in Fig. 5). It should be noted that the three groups of patients were constructed to provide well diagnosed cases. In the clinical daily life, no doubt that patients with mixed structures could occur. This will be easily assessed by using the probability $P_{111111}$.

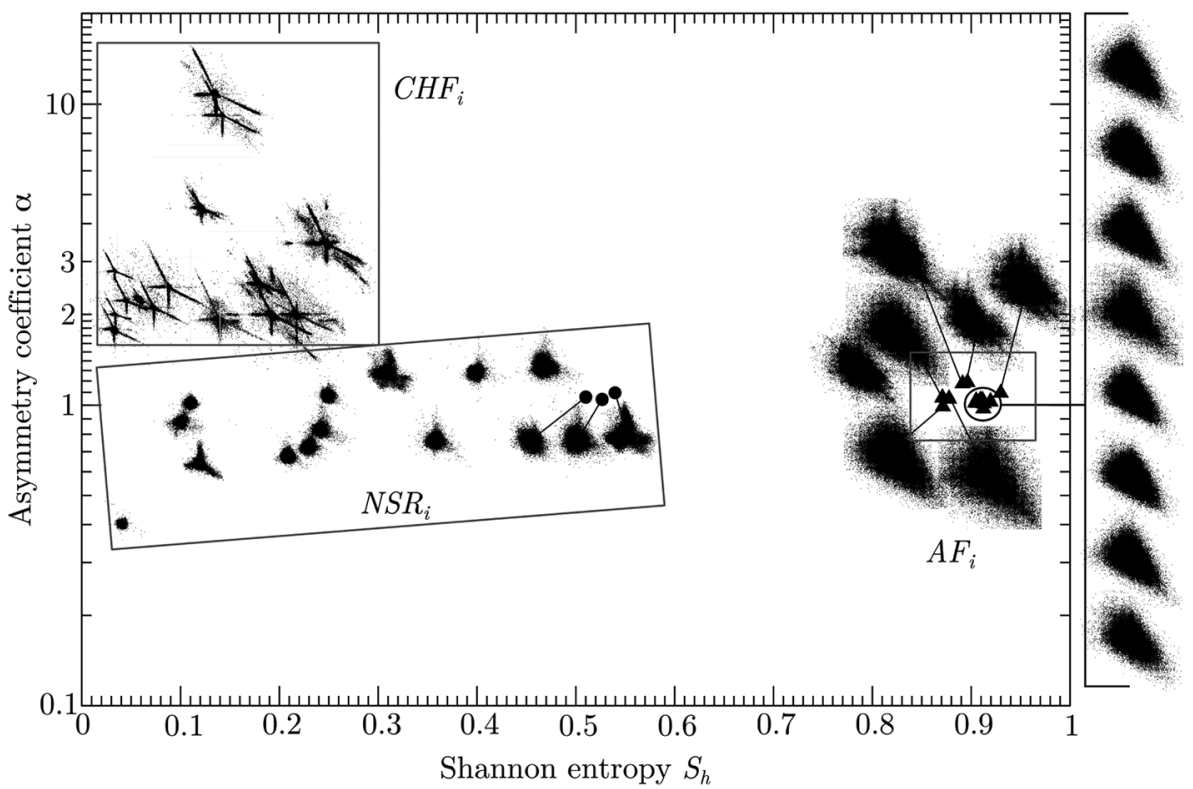

FIG. 5. Map spanned by the relative Shannon entropy $S_{h}$ and the asymmetry coefficient $\alpha$. The 15 patients of each group are reported. A logarithmic scale is used in the ordinate axis to improve the clarity of the map. 
The relative Shannon entropy was rather small for the CHF group, significantly greater for healthy patients, and yet substantially greater for the AF group than for healthy patients (Table II). The asymmetry coefficient $\alpha$ was close to 1 for healthy subjects and patients with AF and significantly greater for the CHF group thus confirming results obtained in Refs. 16 and 41 . The realization probability $P_{111111}$ (using the three-symbol dynamics) was large for patients with CHF, significantly smaller in healthy patients, and again significantly smaller for the AF group than for the healthy subjects. The values of the characteristic time scale $T_{c}$ of the cardiac dynamics were significantly different between the three groups: this characteristics time scale was small for patients with CHF, greater for healthy subjects and yet greater for patients with AF. This result is illustrated by the evolution of the Shannon entropy versus the time threshold $\tau_{v a r}$ used to define the two-symbol symbolic dynamics (Fig. 6).

Since associated with a small Shannon entropy and a small characteristic time scale, the cardiac dynamics of patients affected by congestive heart failure is characterized by a decreased sinus variability. These patients have the largest percentage of sequence "111111," a feature that reveals a poor adaptation capacity of the heart. This is consistent with the small central disk observed in the first-return maps (see Fig. 3, for instance). A decreased cardiac variability in these patients can be indicative of an increased risk of sudden cardiac death, as shown by Ponikowski et al. ${ }^{53} \mathrm{CHF}$ is also associated with large beat-to-beat fluctuations that we identified as being VPCs from the typical structure in four segments of the first-return map. Their presence is associated with an increased asymmetry coefficient $\alpha$, since VPCs

TABLE II. Mean and standard deviation of the relative Shannon entropy $S_{h}$, of the realization probability of sequence " 111111 " encoded with the 3 -symbol dynamics, of the asymmetry coefficient $\alpha$, and of the characteristic time scale $T_{c}$ computed from the 2-symbol dynamics, for each group of the Physionet patients. $p$-values between each group are also reported (Wilcoxon rank-sum test).

\begin{tabular}{|c|c|c|}
\hline Patients & $\overline{S_{h}}$ & $p$-value \\
\hline $\begin{array}{l}\mathrm{NSR}_{i} \\
\mathrm{CHF}_{i} \\
\mathrm{AF}_{i}\end{array}$ & $\begin{array}{l}0.294 \pm 0.166 \\
0.116 \pm 0.071 \\
0.893 \pm 0.035\end{array}$ & $\left.\begin{array}{l}] p<0.01 \\
] p<0.01\end{array}\right] p<0.01$ \\
\hline Patients & $\bar{\alpha}$ & $p$-value \\
\hline $\begin{array}{l}\mathrm{NSR}_{i} \\
\mathrm{CHF}_{i} \\
\mathrm{AF}_{i}\end{array}$ & $\begin{array}{l}0.951 \pm 0.275 \\
3.472 \pm 2.770 \\
1.068 \pm 0.106\end{array}$ & $\left.\begin{array}{l}] p<0.01 \\
] p<0.01\end{array}\right] p=0.52$ n.s. \\
\hline Patients & $\overline{111111}$ & $p$-value \\
\hline $\begin{array}{l}\mathrm{NSR}_{i} \\
\mathrm{CHF}_{i} \\
\mathrm{AF}_{i}\end{array}$ & $\begin{array}{c}69.56 \pm 17.36 \% \\
87.99 \pm 8.03 \% \\
1.52 \pm 4.95 \%\end{array}$ & $\left.\begin{array}{l}] p<0.01 \\
] p<0.01\end{array}\right] p<0.01$ \\
\hline Patients & $\overline{T_{c}}$ & $p$-value \\
\hline $\begin{array}{l}\mathrm{NSR}_{i} \\
\mathrm{CHF}_{i} \\
\mathrm{AF}_{i}\end{array}$ & $\begin{array}{c}17.07 \pm 5.95 \mathrm{~ms} \\
5.87 \pm 2.13 \mathrm{~ms} \\
115.07 \pm 28.27 \mathrm{~ms}\end{array}$ & $\left.\begin{array}{l}] p<0.01 \\
] p<0.01\end{array}\right] p<0.01$ \\
\hline
\end{tabular}

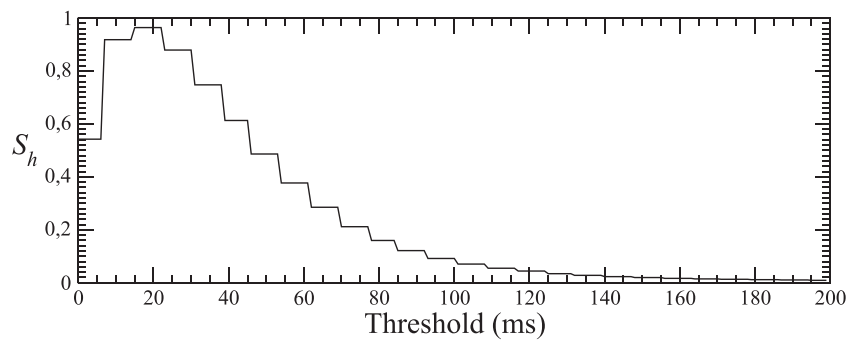

(a)NSR 13 patient: $T_{c}=16 \mathrm{~ms}$

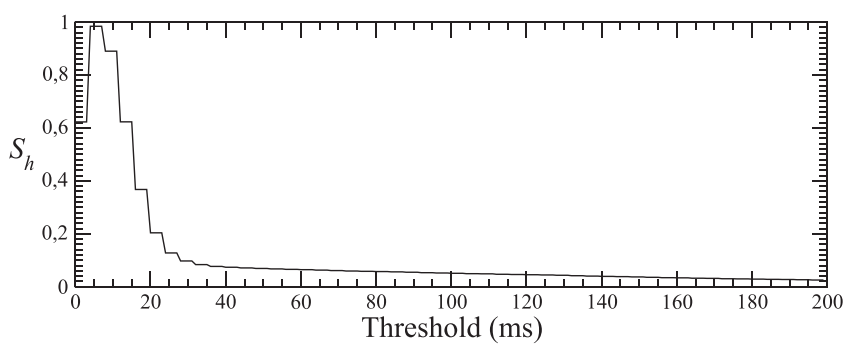

(b) $\mathrm{CHF}_{7}$ patient: $T_{c}=5 \mathrm{~ms}$

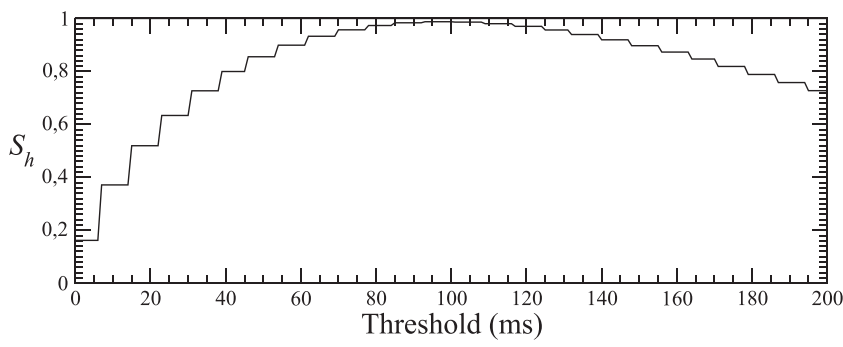

(c) $\mathrm{AF}_{13}$ patient: $T_{c}=94 \mathrm{~ms}$

FIG. 6. Evolution of the relative Shannon entropy computed from the 2 -symbol dynamics versus the time threshold $\tau_{v a r}$ for three patients. The characteristic time scale $T_{c}$ corresponds to the time threshold at which the entropy is the largest.

induce an imbalance between accelerations and decelerations in the cardiac rhythm.

Patients affected by atrial fibrillation present the largest Shannon entropy and a large characteristic time scale. It reveals a wide complexity of the heart rhythm, in agreement with the large domain visited by the first-return maps. The percentage of sequence " 111111 " is very small and the asymmetry coefficient $\alpha$ is close to 1 : this is indicative of many large beat-to-beat fluctuations in the heart rate, with accelerations and decelerations.

Subjects with normal sinus rhythm present an intermediate Shannon entropy (around 0.3) and an asymmetry coefficient $\alpha$ close to 1 . Indeed, a healthy heart has a good adaptation capacity leading to small and isolated beat-tobeat variations in the heart rate. These subjects own a moderate cardiac variability as revealed by the characteristic time scale of the cardiac dynamics (Fig. 6(a)).

\section{Blind validation}

To test the prospective discrimination power of our technique, and since the criteria used to classify the patients in three groups were determined from a retrospective study, we 
TABLE III. Relative Shannon entropy $S_{h}$, asymmetry coefficient $\alpha$, realization probability of sequence " 111111 " encoded with the 3 -symbol dynamics, and characteristic time scale $T_{c}$ computed from the 2-symbol dynamics, for the 15 additional patients selected from the Physionet database, Threshold values: $S_{c}=0.66$ and $\alpha_{c}=1.5$.

\begin{tabular}{|c|c|c|c|c|c|c|c|c|}
\hline \# & $S_{h}$ & $\alpha$ & $\begin{array}{c}P_{111111} \\
(\%)\end{array}$ & $\begin{array}{c}T_{c} \\
(\mathrm{~ms})\end{array}$ & $\begin{array}{l}\text { NSR } \\
S_{h}<S_{c} \\
\alpha<\alpha_{c}\end{array}$ & $\begin{array}{l}\mathrm{CHF} \\
\alpha \geq \alpha_{c}\end{array}$ & $\begin{array}{l}\mathrm{AF} \\
S_{h} \geq S_{c} \\
\alpha<\alpha_{c}\end{array}$ & $\begin{array}{l}\text { Actual } \\
\text { status }\end{array}$ \\
\hline 1 & 0.86 & 1.15 & 4.6 & 102 & & & $\mathrm{x}$ & $\mathrm{AF}$ \\
\hline 2 & 0.09 & 2.13 & 91.0 & 8 & & $\mathrm{x}$ & & $\mathrm{CHF}$ \\
\hline 3 & 0.11 & 0.55 & 89.1 & 16 & $\mathrm{x}$ & & & NSR \\
\hline 4 & 0.07 & 0.61 & 93.5 & 16 & $\mathrm{x}$ & & & NSR \\
\hline 5 & 0.10 & 0.31 & 90.0 & 16 & $\mathrm{x}$ & & & NSR \\
\hline 6 & 0.91 & 1.00 & 0.8 & 101 & & & $\mathrm{x}$ & $\mathrm{AF}$ \\
\hline 7 & 0.08 & 1.73 & 92.4 & 8 & & $\mathrm{x}$ & & $\mathrm{CHF}$ \\
\hline 8 & 0.90 & 0.98 & 0.9 & 133 & & & $\mathrm{x}$ & $\mathrm{AF}$ \\
\hline 9 & 0.06 & 1.44 & 94.5 & 8 & $\mathrm{x}$ & & & $\mathrm{CHF}$ \\
\hline 10 & 0.18 & 0.84 & 82.7 & 16 & $\mathrm{x}$ & & & NSR \\
\hline 11 & 0.02 & 3.61 & 97.9 & 8 & & $\mathrm{x}$ & & $\mathrm{CHF}$ \\
\hline 12 & 0.90 & 1.01 & 0.9 & 148 & & & $\mathrm{x}$ & $\mathrm{AF}$ \\
\hline 13 & 0.91 & 0.98 & 0.2 & 140 & & & $\mathrm{x}$ & $\mathrm{AF}$ \\
\hline 14 & 0.26 & 2.88 & 71.8 & 9 & & $\mathrm{x}$ & & $\mathrm{CHF}$ \\
\hline 15 & 0.20 & 0.61 & 79.3 & 17 & $\mathrm{x}$ & & & NSR \\
\hline
\end{tabular}

performed a blind validation. We therefore selected 15 additional patients from the Physionet database, 5 corresponding to normal sinus rhythm, 5 to congestive heart failure, and 5 to atrial fibrillation. We then randomized them, erasing the knowledge about their actual status. We used our technique to classify them. Results are reported Table III with the criteria retained for defining the three groups.

According to our criteria based on a threshold value for the Shannon entropy $\left(S_{c}=0.66\right)$ and for the asymmetry coefficient $\left(\alpha_{c}=1.5\right), 14$ patients were correctly classified (accuracy $=93.3 \%$, sensitivity $=90 \%$, and specificity $=100 \%$ ) when only these two biomarkers are used.

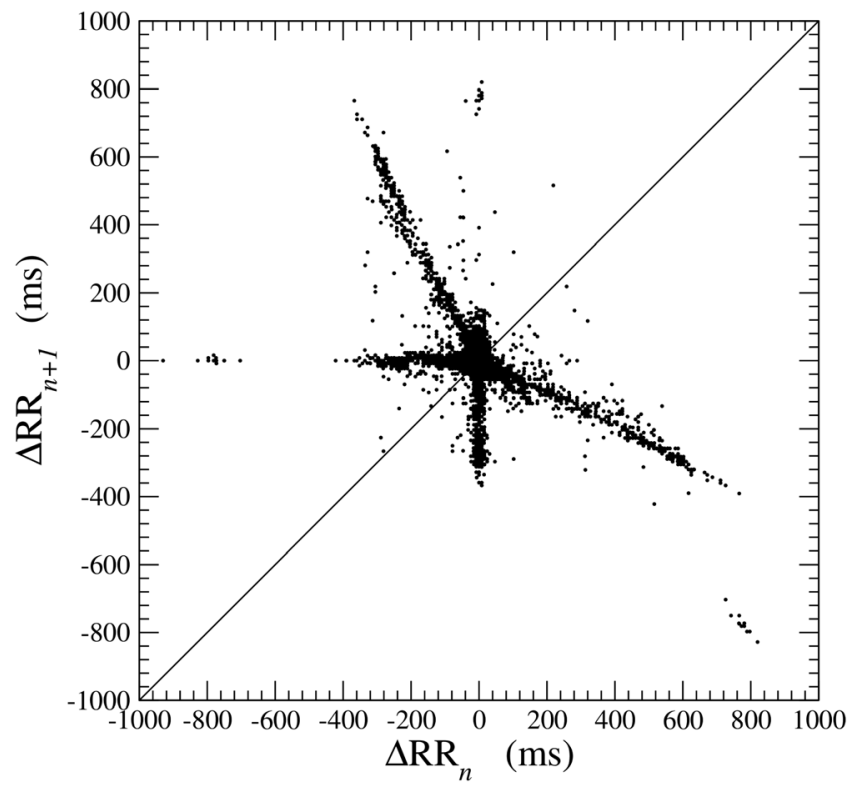

FIG. 7. First-return map built on $\Delta$ RR for patient 9 of additional patients used for the prospective validation of our technique. The four segments characteristics of patients with congestive heart failure are clearly observed in this map.
Nevertheless, patient 9 has an asymmetry coefficients equal to 1.44 , that is, to a value very close to the threshold $\alpha_{c}$. A further investigation would be recommended in such a case. If the characteristic time scale $T_{c}$ is considered, it is found that patient 9 has a time scale $(8 \mathrm{~ms})$ equal to half the time scale obtained for the patients with normal sinus rhythm (16 ms) and equal to $T_{c}$ found in patients with congestive heart failure $(8 \mathrm{~ms})$. The probability $P_{111111}$ cannot discriminate these two groups. Moreover, the first-return map (Fig. 7) clearly presents the four segments characteristic of patients with congestive heart failure. Patient 9 is therefore characterized by one biomarker very close to the threshold value but a quick additional analysis allows to easily provide the right diagnostic.

\section{CONCLUSION}

By investigating three groups of adults with clearly identified status as reported in the Physionet database, we showed that each group was characterized by a first-return map built from the $\Delta \mathrm{RR}$ with a specific structure. Firstreturn maps built on the $\Delta \mathrm{RR}$ recorded in adults with normal sinus variability, atrial fibrillation, and congestive heart failure presented a small central cloud, a quite extended triangular shape, and oriented segments, respectively. We investigated long duration time series (recorded for few hours) and focused our attention on the global dynamics rather than on short duration episodes of arrhythmias. Our two biomarkers, namely, a Shannon entropy for quantifying the complexity of the dynamics (based on a symbolic dynamics with an age-dependent partition) and an asymmetry coefficient for quantifying the balance between fast accelerations and fast decelerations in the rhythm, allowed us to automatically group the first-return maps according to their structure, that is, to discriminate these three groups of patients. We here proposed two threshold values for these two biomarkers to define domains in the plot spanned by the Shannon entropy and the asymmetry coefficient corresponding to normal sinus rhythm, congestive heart failure, and atrial fibrillation, respectively. These threshold values were found reliable for screening patients with very well defined cardiac profiles. A validation was then performed with additional patients who were not used in the retrospective analysis for determining the threshold values. For a more refined validation, this first analysis should be completed by a clinical protocol with diagnosis provided by physicians.

\section{ACKNOWLEDGMENTS}

Ph.D. theses of E. Fresnel and A. Kerfourn are supported by ADIR Association. Ph.D. thesis of E. Yacoub was supported by "La Fondation de France."

${ }^{1}$ Task Force of the European Society of Cardiology and the North American Society of Pacing and Electrophysiology, "Heart rate variability: standards of measurement, physiological interpretation, and clinical use," Circulation 93, 1043-1065 (1996).

${ }^{2}$ J. P. Saul, Y. Arai, R. D. Berger, L. S. Lilly, W. S. Colucci, and R. J. Cohen, "Assessment of autonomic regulation in chronic congestive heart failure by heart rate spectral analysis," Am. J. Cardiol. 61, 1292-1299 (1988). 
${ }^{3}$ B. M. Szabò, D. J. van Veldhuisen, J. Brouwer, J. Haaksma, and K. I. Lie, "Relation between severity of disease and impairment of heart rate variability parameters in patients with chronic congestive heart failure secondary to coronary artery disease," Am. J. Cardiol. 76, 713-716 (1995).

${ }^{4} J$. Nolan, A. D. Flapan, S. Capewell, T. M. MacDonald, J. M. Neilson, and D. J. Ewing, "Decreased cardiac parasympathetic activity in chronic heart failure and its relation to left ventricular function," Br. Heart J. 67, 482-485 (1992).

${ }^{5}$ D. J. Ewing, I. W. Campbell, and B. F. Clarke, "Assessment of cardiovascular effects in diabetic neuropathy and prognostic implications," Ann. Intern. Med. 92, 308-311 (1980).

${ }^{6}$ R. E. Kleiger, J. P. Miller, J. T. Bigger, and A. J. Moss, "Decreased heart rate variability and its association with increased mortality after acute myocardial infarction,” Am. J. Cardiol. 59, 256-262 (1987).

${ }^{7} \mathrm{~A}$. Babloyantz and A. Destexhe, "Is the normal heart a periodic oscillator?" Biol. Cybern. 58, 203-211 (1988).

${ }^{8}$ A. Voss, J. Kurths, H. J. Kleiner, A. Witt, N. Wessel, P. Saparin et al., "The application of methods of non-linear dynamics for the improved and predictive recognition of patients threatened by sudden cardiac death," Cardiovasc. Res. 31, 419-433 (1996).

${ }^{9}$ N. Wessel, C. Ziehmann, J. Kurths, U. Meyerfeldt, A. Schirdewan, and A. Voss, "Short-term forecasting of life-threatening cardiac arrhythmias based on symbolic dynamics and finite-time growth rates," Phys. Rev. E 61, 733-739 (2000).

${ }^{10}$ G. Schmidt and G. E. Monfill, "Nonlinear methods for heart rate variability assessment," in Heart Rate Variability, edited by M. Malik and A. J. Camm (Futura, Armonk, NY, 1995), pp. 87-98.

${ }^{11}$ N. Levinson, "A second order differential equation with singular solutions," Ann. Math. 50, 127-153 (1949).

${ }^{12} \mathrm{P}$. Collet and J. P. Eckmann, Iterated Maps on the Interval as Dynamical Systems (Birkhauser Boston, 1980).

${ }^{13}$ B.-L. Hao, Elementary Symbolic Dynamics and Chaos in Dissipative Systems (World Scientific, 1989).

${ }^{14} \mathrm{~N}$. Wessel, M. Riedl, and J. Kurths, "Is the normal heart rate 'chaotic' due to respiration," Chaos 19, 028508 (2009).

${ }^{15}$ F. Perticone, R. Ceravolo, R. Maio, C. Cosco, and P. L. Mattioli, "Heart rate variability and sudden infant death syndrome," Pacing Clin. Electrophysiol. 13, 2096-2099 (1990).

${ }^{16}$ U. S. Freitas, E. Roulin, J.-F. Muir, and C. Letellier, "Identifying chaos from heart rate: The right task?” Chaos 19, 028505 (2009).

${ }^{17} \mathrm{~L}$. Glass, "Introduction to controversial topics in nonlinear science: Is the normal heart rate chaotic?" Chaos 19, 028501 (2009).

${ }^{18}$ J. Q. Zhang, A. V. Holden, O. Monfredi, M. R. Boyett, and H. Zhang, "Stochastic vagal modulation of cardiac pacemaking may lead to an erroneous identification of cardiac chaos," Chaos 19, 028509 (2009).

${ }^{19}$ J. Alvarez-Ramirez, E. Rodriguez, and J. C. Echeverria, "Delays in the human heartbeat dynamics," Chaos 19, 028502 (2009).

${ }^{20}$ C. Letellier, G. G. Rodrigues, J.-F. Muir, and L. A. Aguirre, "Individuality of breathing patterns in patients under noninvasive mechanical ventilation evidenced by chaotic global models," Chaos 23(1), 013137 (2013).

${ }^{21}$ J. P. Saul, R. F. Rea, D. L. Eckberg, R. D. Berger, and R. J. Cohen, "Heart rate and muscle sympathetic nerve variability during reflex changes of autonomic activity," Am. J. Physiol. 258, H713-H721 (1990).

${ }^{22}$ A. Malliani, "Cardiovascular sympathetic afferent fibers," Rev. Physiol., Biochem. Pharmacol. 94, 11-74 (1982).

${ }^{23}$ A. Malliani, M. Pagani, F. Lombardi, and S. Cerutti, "Cardiovascular neural regulation explored in the frequency domain," Circulation 84, 482-492 (1991).

${ }^{24}$ A. Rosenblueth and F. A. Simeone, "The interrelations of vagal and accelerator effects on the cardiac rate," Am. J. Physiol. 31, 42-55 (1934).

${ }^{25}$ A. D. Jose and D. Collison, "The normal range and determinants of the intrinsic heart rate in man," Cardiovasc. Res. 4, 160-167 (1970).

${ }^{26}$ H. Tanaka, K. D. Monahan, and D. R. Seals, "Age-predicted maximal heart rate revisited," J. Am. Coll. Cardiol. 37, 153-156 (2001).

${ }^{27}$ T. J. Ingall, J. G. McLeod, and P. C. O'Brien, “The effect of ageing on autonomic nervous system function," Aust. N. Z. J. Med. 20, 570-577 (1990).

${ }^{28}$ K. Umetani, D. H. Singer, R. McCraty, and M. Atkinson, "Twenty-four hour time domain heart rate variability and heart rate: Relations to age and gender over nine decades," J. Am. Coll. Cardiol. 31, 593-601 (1998).

${ }^{29}$ K. Jensen-Urstad, N. Storck, F. Bouvier, M. Ericson, L. E. Lindblad, and M. Jensen-Urstad, "Heart rate variability in healthy subjects is related to age and gender," Acta Physiol. Scand. 160, 235-241 (1997).
${ }^{30} \mathrm{P}$. K. Stein, R. E. Kleiger, and J. N. Rottman, "Differing effects of age on heart rate variability in men and women," Am. J. Cardiol. 80, 302-305 (1997).

${ }^{31}$ D. Ramaekers, H. Ector, A. E. Aubert, A. Rubens, and F. Van de Werf, "Heart rate variability and heart rate in healthy volunteers," Eur. Heart J. 19, 1334-1341 (1998).

${ }^{32}$ C. Vallbona, M. M. Desmond, A. J. Rudolph, L. F. Pap, R. M. Hill, R. R. Franklin, and J. B. Rush, "Cardiodynamic studies in the newborn. II. Regulation of the heart rate," Biol. Neonat. 5, 159-199 (1963).

${ }^{33}$ J. P. Finley and S. T. Nugent, "Heart rate variability in infants, children and young adults," J. Auton. Nerv. Syst. 51, 103-109 (1995).

${ }^{34} \mathrm{M}$. Massin and G. von Bernuth, "Normal ranges of heart rate variability during infancy and childhood," Pediatr. Cardiol. 18, 297-302 (1997).

${ }^{35}$ O. V. Korkushko, V. B. Shatilo, Yu. I. Plachinda, and T. V. Shatilo, "Autonomic control of cardiac chronotropic function in man as a function of age: Assessment by power spectral analysis of heart rate variability," J. Auton. Nerv. Syst. 32, 191-198 (1991).

${ }^{36} \mathrm{~K}$. Tateno and L. Glass, "Automatic detection of atrial fibrillation using the coefficient of variation and density histograms of RR and deltaRR intervals," Med. Biol. Eng. Comput. 39, 664-671 (2001).

${ }^{37}$ C. Huang, S. Ye, H. Chen, D. Li, F. He, and Y. Tu, "A novel method for detection of the transition between atrial fibrillation and sinus rhythm," IEEE Trans. Biomed. Eng. 58(4), 1113-1119 (2011).

${ }^{38}$ J. Lian, L. Wang, and D. Muessig, "A simple method to detect atrial fibrillation using RR intervals,” Am. J. Cardiol. 107(10), 1494-1497 (2011).

${ }^{39}$ D. E. Ritscher and S. Sarkar, "Algorithms for detecting atrial arrhythmias from discriminatory signatures of ventricular cycle lengths," Medtronic Inc., patent 20040092836 (2002).

${ }^{40}$ S. Sarkar, D. Ritscher, and R. Mehra, "A detector for a chronic implantable atrial tachyarrhythmia monitor," IEEE Trans. Biomed. Eng. 55(3), 1219-1224 (2008).

${ }^{41}$ D. D. McManus, J. Lee, O. Maitas, N. Esa, R. Pidikiti, A. Carlucci, J. Harrington, E. Mick, and K. H. Chon, "A novel application for the detection of an irregular pulse using an iPhone $4 \mathrm{~S}$ in patients with atrial fibrillation," Heart Rhythm 10(3), 315-319 (2013).

${ }^{42}$ K. Hnatkova, X. Copie, A. Staunton, and M. Malik, "Numeric processing of Lorenz plots of R-R intervals from long-term ECGs. Comparison with time-domain measures of heart rate variability for risk stratification after myocardial infarction," J. Electrocardiol. 28, 74-80 (1995).

${ }^{43}$ C. E. Shannon, "A mathematical theory of communication," Bell Syst. Tech. J. 27, 379-423 (1948).

${ }^{44}$ P. Guzik, J. Piskorski, T. Krauze, A. Wykretowicz, and H. Wysocki, "Heart rate asymmetry by Poincaré plots of RR intervals," Biomed. Tech. 51(4), 272-275 (2006).

${ }^{45}$ J. Piskorski and P. Guzik, "Geometry of the Poincaré plot of RR intervals and its asymmetry in healthy adults," Physiol. Meas. 28, 287-300 (2007).

${ }^{46}$ J. Piskorski and P. Guzik, "The structure of heart rate asymmetry: Deceleration and acceleration runs," Physiol. Meas. 32, 1011-1023 (2011).

${ }^{47}$ N. Wessel, A. Voss, H. Malberg, C. Ziehmann, H. U. Voss, A. Schirdewan, U. Meyerfeldt, and J. Kurths, "Nonlinear analysis of complex phenomena in cardiological data," Herzschr Elektrophys. 11, 159-173 (2000).

${ }^{48}$ A. Voss, K. Hnatkova, N. Wessel, J. Kurths, A. Sander, A. Schirdewan, A. J. Camm, and M. Malik, "Multiparametric analysis of heart rate variability used for risk stratification among survivors of acute myocardial infarction," Pacing Clin. Electrophysiol. 21, 186-192 (1998).

${ }^{49}$ A. L. Goldberger, L. A. N. Amaral, L. Glass, J. M. Hausdorff, P. Ch. Ivanov et al., "PhysioBank, PhysioToolkit, and PhysioNet: Components of a new research resource for complex physiologic signals," Circulation 101, e215-e220 (2000).

${ }^{50}$ R. Langendorf, "Ventricular premature systoles with postponed compensatory pause," Am. Heart J. 46, 401-404 (1953).

${ }^{51}$ S. K. Agarwal, R. J. Simpson, P. Rautaharju, A. Alfonso, E. Shahar et al., "Relation of ventricular premature complexes to heart failure," Am. J. Cardiol. 109, 105-109 (2012).

${ }^{52}$ E. Patterson and B. J. Scherlag, "Decremental conduction in the posterior and anterior AV nodal inputs," J. Intervent. Cardiac Electrophysiol. 7, 137-148 (2002).

${ }^{53}$ P. Ponikowski, S. D. Anker, T. P. Chua, R. Szelemej et al., "Depressed heart rate variability as an independent predictor of death in chronic congestive heart failure secondary to ischemic or idiopathic dilated cardiomyopathy," Am. J. Cardiol. 79, 1645-1650 (1997). 